\title{
Outcomes and computed tomography radiomic features extraction in soft tissue sarcomas treated with neoadjuvant radiation therapy
}

\author{
Javier González-Viguera ${ }^{1}$, Gabriel Reynés-Llompart ${ }^{2}$, Alicia Lozano ${ }^{1}$ \\ ${ }^{1}$ Catalan Institute of Oncology, Radiation Oncology, L'Hospitalet de Llobregat, Spain \\ ${ }^{2}$ Catalan Institute of Oncology, Medical Physics and Radiological Protection Service, L'Hospitalet de Llobregat, Spain
}

\begin{abstract}
Background: The aim of the study was to evaluate the management, toxicity and treatment responses of patients treated with neoadjuvant radiotherapy (NART) for soft tissue sarcomas (STS) and to analyse the potential of radiomic features extracted from computed tomography (CT) scans.

Materials and methods: This is a retrospective and exploratory study with patients treated between 2006 and 2019. Acute and chronic toxicities are evaluated. Local progression free survival (LPFS), distant progression free survival (DPFS) and overall survival (OS) are analysed. Radiomic features are obtained.

Results: A total of 25 patients were included. Median follow-up is 24 months. Complications in surgical wound healing were observed in $20 \%$ of patients, chronic fibrosis was documented as grade 1 (12\%) and grade 2 (12\%) without grade 3 events and chronic lymphedema as grade 1 (8\%) and grade 2 (20\%) without grade 3 events. Survival variables were LPFS 76\%, DPFS 62\% and OS 67.2\% at 2-year follow-up. CT radiomics features were associated significantly with local control (GLCM-correlation), systemic control (HUmin, HUpeak, volume, GLCM-correlation and GLZLM-GLNU) and OS (GLZLM-SZE).

Conclusions: STS treated with NART in our centre associate with an OS and toxicity comparable to other series. CT radiomic features have a prognosis potential in STS risk stratification. The results of our study may serve as a motivation for future prospective studies with a greater number of patients.
\end{abstract}

Key words: soft tissue sarcomas; neoadjuvant radiotherapy; radiomic features

Rep Pract Oncol Radiother 2021;26(5):804-813

\section{Introduction}

Soft tissue sarcomas (STS) (List of abbreviations is included in Supplementary File - Table S1) are a large and heterogeneous group of tumours of mesenchymal origin. It is a rare entity, representing less than $1 \%$ of all adult solid malignancies and almost $21 \%$ of all paediatric solid malignancies. STS are characterised by the complexity of their multidisciplinary management $[1,2]$.

There are more than 70 different histological subtypes of STS [3], they can be presented at any age and they are not restricted to a specific location in the body. The rarity of the disease combined with the diverse number of histological subtypes and locations results in a complexity of managing STS.

Address for correspondence: Javier González-Viguera, Catalan Institute of Oncology — Hospital Duran i Reynals. Avinguda de la Gran Via de L’Hospitalet, 199-203, 08908. L’Hospitalet de Llobregat, Barcelona, Spain; e-mail: jgonzalezv@iconcologia.net 
Treatment and clinical outcomes after treatment differ greatly depending on the histological subtype and grade, the anatomical location of the primary tumour and the occurrence of metastatic tumour disease. For localised high-grade STS, conservative treatment is currently proposed, usually involving a wide excision in combination with radiotherapy (RT) and chemotherapy (ChT), either neoadjuvant or adjuvant [4].

The strongest evidence regarding the sequence of treatment is found in the phase III multicentre trial by O'Sullivan and colleagues [5]. The main objective of the study was to determine whether the sequence of treatment is related to a major complication in surgical wound healing. The results of the study determined that neoadjuvant RT versus adjuvant RT offers several advantages, including lower total radiation doses, smaller target volumes and lower late toxicity. In particular, chronic complications (oedema and fibrosis) were more common after adjuvant RT. However, greater difficulty in surgical wound healing was documented in the neoadjuvant RT group ( $35 \%$ vs. $17 \%)$.

There is also some evidence of increased overall survival (OS) in favour of neoadjuvant RT. In a meta-analysis [6] the average survival was $76 \%$ $(62-88 \%)$ in the preoperative group and $67 \%$ $(41-83 \%)$ in the postoperative group. In a retrospective study [7], neoadjuvant RT was associated with a statistically significant improvement in OS compared to adjuvant RT (hazard ratio $=0.72$, $\mathrm{p}<0.01$ ). However, these findings should be interpreted with caution due to heterogeneity and bias in the available studies.

In recent years there have been advances in the knowledge of the molecular biology of STS. However, despite major research efforts, molecular alterations have not yet been established as prognostic markers and therapeutic decisions are still mainly made using basic clinical-pathological information, such as TNM classification. Therefore, treatment has evolved more discreetly and the improvement in clinical outcomes has not been as remarkable: in contrast to the high local control rates achieved with current therapeutic regimens (up to $94 \%$ at 5 years), systemic control and overall survival rates are comparatively low $(61 \%$ and $64 \%$ at 5 years, respectively) [8].

Over the last decades, numerous studies have evaluated the incorporation of nomograms into clinical practice to personalize risk assessment and individualize therapeutic decision making [9]. Similarly, quantitative analysis of radiological features or Radiomics offers great potential as a non-invasive tool to complement existing diagnostic and treatment methods. In Oncology, Radiomics has been associated with histological grade, response to treatment and prognosis, and in the future this discipline could allow the construction of predictive models that help in decision making in clinical practice [10].

In this study, a data research of patients diagnosed with STS treated with neoadjuvant RT is performed, with the aim of:

- evaluating the therapeutic management, side effects and treatment responses of patients treated in our centre;

- evaluating radiomic features extracted from planning CT scans as prognostic factors prior to treatment.

\section{Materials and methods}

This is a retrospective, exploratory, analytical and longitudinal study. The sample size is made up of patients diagnosed with STS with indication of neoadjuvant RT, treated between 2006 and 2019 in our centre. At the time of the statistical analysis, all patients under study with intention to treat are included.

The baseline characteristics of the patients under study include gender, age at diagnosis, location of the lesion (limb, trunk or head and neck), histological grade according to the Fédération Nationale des Centres de Lutte Contre le Cancer (FNCLCC), histological type by biopsy defined by the World Health Organization (WHO) [3] and clinical stage according to the seventh and eighth editions of the American Joint Committee on Cancer (AJCC) [11].

The RT procedure is performed using a linear accelerator (Varian models). The acquisition of the planning CT images is obtained by using a CT scan machine (Philips model) and is acquired every 3 $\mathrm{mm}$ in a reproducible way by using immobilizers. The RT technique (3DCRT, VMAT or IMRT) depends on the location of the target lesion in order to respect, as far as possible, the full circumference of the limb and to achieve a minimum dose of at least $95 \%$ and a maximum acceptable dose of $107 \%$ of the total prescribed. 
For the delimitation of the treatment volume in the preoperative act, the gross tumour volume (GTV) is defined on T1 sequences with gadolinium from the magnetic resonance imaging (MRI); the clinical target volume (CTV1) includes the GTV with an additional margin ( $40 \mathrm{~mm}$ in length and 15 $\mathrm{mm}$ radially) limited by the affected compartment and including the peritumoral oedema defined on T2 sequences of the MRI, and the planning target volume (PTV1) includes the CTV1 with an isotropic margin of 5-10 $\mathrm{mm}$. The prescription dose of neoadjuvant RT is 50 Gy at 2 Gy per fraction per day over PTV1, concurrent with ChT.

After neoadjuvant RT, surgery is based on a wide tumour excision, reserving amputation for cases of local complications or when complete tumour resection with safety margins is not possible. Following surgery, an analysis of the surgical specimen is performed, obtaining the definitive FNCLCC grade and histological type according to the WHO classification, the pathological stage corresponding to the AJCC system, the percentage of necrosis, the resection margins (R0, R1 or R2) and possible stage decreasing after RT.

Acute post-surgical complications and total days at hospital are evaluated as a surrogate variable of surgical wound healing difficulty.

In cases of affected margins after surgery $(\mathrm{R} 1$ and $\mathrm{R} 2$ ), additional treatment of the surgical bed with adjuvant RT is assessed. For the treatment volume delimitation in the postoperative act, a high risk area is defined in the planning CT images (corresponding to the area of post-surgical changes and metal clips placed in the surgical act, always considering the macroscopic tumour volume defined on the preoperative MRI in T1 sequences, and the possible areas of dissemination: skin scar, surgical drainage path, haematomas and lymphoceles); the boost clinical target volume (CTV2) includes the high-risk area with an additional margin $(40 \mathrm{~mm}$ in length and $15 \mathrm{~mm}$ radially) limited by the affected compartment, and the boost planning target volume (PTV2) includes the CTV2 with an isotropic margin of 5-10 $\mathrm{mm}$. The prescription dose of adjuvant RT is 16 Gy at 2 Gy per fraction per day over the PTV2.

The acute and chronic toxicities of RT are evaluated in detail during the treatment period and during the oncological follow-up. Adverse effects to $\mathrm{RT}$ are assessed according to the Common Termi- nology Criteria for Adverse Events (CTCAE v5.0) [12]. The adverse effects considered are dermitis and lymphoedema as acute toxicity and fibrosis, lymphoedema, pathological fracture and functional limitation as chronic toxicity. Likewise, other relevant toxicities are reported in each case.

Oncological follow-up consists of periodic local and systemic evaluations. Generally, local assessment is based on a physical examination and an MRI of the treated region on a six-monthly schedule during the first year and on an annual schedule until the completion of five years from treatment. Due to the avidity of STS for lung spread [1], systemic assessment is performed with the practice of a chest radiography every three months during the first two years and every six months until completing five years from treatment (in case of doubt, the lesions are checked with a chest CT for confirmation). Local and systemic control rates, local (LPFS) and distant (DPFS) progression free survivals, overall survival (OS) and the patient's status at the last control (alive free of relapse, alive after local relapse, alive after systemic relapse, alive after local and systemic relapse, dead due to disease and dead from other causes).

Regarding the study of prognostic factors based on Radiomics, the volume of interest (VOI) corresponds to the GTV volumes segmented in the planning CT of neoadjuvant RT. All the pre- processing and radiomic feature extraction steps are performed using LIFEx software (version 6.0) [13]. LIFEx software performs a direct calculation of the radiomic features of each VOI from every CT uploaded to the software.

The radiomics characteristics analysed in this study are shape, first order and second order variables obtainable through LIFEx, mentioned below (List of extracted radiomics features is included in Supplementary File - Table S2):

- shape variables: they quantify the geometric shape of the VOI. The following are analysed: volume, sphericity and compacity;

- first order variables: they take into account the distribution of pixel intensities and the use of elementary metrics to calculate geometric characteristics. They are derived from a single histogram generated from the $2 \mathrm{D}$ region or from the entire 3D volume. The following are analysed: skewness, kurtosis, excess kurtosis, entropy, energy and other conventional variables (mean, 
minimum, maximum, standard deviation and average intensity peaks in a 0.5 and $1 \mathrm{~mL}$ sphere);

- second order variables: they take into account texture characteristics and relationships between pixels to model intratumoral heterogeneity. Texture characteristics are generated from different descriptive matrices. We include these:

- grey level co-occurrence matrix (GLCM). It is a matrix that presents the number of times two levels of intensity have occurred at two pixels with a specific distance. Homogeneity, energy, contrast, correlation, entropy and dissimilarity are analysed,

- grey level run length matrix (GLRLM). This is a matrix that shows the length of consecutive pixels having the same intensity. The following are analysed: SRE, LRE, LGRE, HGRE, SRLGE, SRHGE, LRLGE, LRHGE, GLNU, RLNU and RP,

- grey level zone length matrix (GLZLM). This matrix considers the size of the homogenous zones in each dimension. SZE, LZE, LGZE, HGZE, SZLGE, SZHGE, LZLGE, LZHGE, GLNU, ZLNU and ZP are analysed,

- neighbourhood grey level different matrix (NGLDM). This is a matrix that considers the intensities of the neighbouring pixels rather than the pixel itself. Coarseness, contrast and busyness are analysed.

A descriptive and survival analysis is performed using the SPSS software (v25.0) [14] and the radiomics variables are tested together with clinical-pathological variables in the PyRadiomics software (v3.0) [15] to analyse their predictive power using Student's T-test and the Wilcoxon's test, considering a $\mathrm{p}$ value below 0.05 to be significant. The associations in the radiomics study analysed are local and systemic control and overall survival.

\section{Results}

The data obtained in the study are shown in Table 1. A total of 25 patients were analysed, 13 women (52\%) and 12 men (48\%). The average age at diagnosis was 50.5 years ( $27-75$ years).

The limbs were the most frequent location of tumourogenesis (92\%), with predominance of the lower extremities. The remaining cases occurred at the trunk (4\%) and head and neck (4\%).

From the analysis of the samples obtained by biopsy, the following histological subtypes were
Table 1. Summary of the results of the study

\begin{tabular}{|c|c|}
\hline \multicolumn{2}{|l|}{ Patients } \\
\hline Number of patients & 25 \\
\hline \multicolumn{2}{|l|}{ Gender } \\
\hline Women & $13(52 \%)$ \\
\hline Men & $12(48 \%)$ \\
\hline Average age & 50.5 years $(27-75)$ \\
\hline \multicolumn{2}{|l|}{ Clinical information at diagnosis } \\
\hline \multicolumn{2}{|l|}{ Site } \\
\hline Limbs & $23(92 \%)$ \\
\hline Lower limbs & $14(56 \%)$ \\
\hline Upper limbs & $9(36 \%)$ \\
\hline Trunk & $1(4 \%)$ \\
\hline Head and neck & $1(4 \%)$ \\
\hline \multicolumn{2}{|l|}{ Histological type by biopsy } \\
\hline Fusocellular sarcoma & $9(36 \%)$ \\
\hline Synovial sarcoma & $4(16 \%)$ \\
\hline Pleomorphic sarcoma & $3(12 \%)$ \\
\hline Myxoid sarcoma & $2(8 \%)$ \\
\hline Leiomyosarcoma & $2(8 \%)$ \\
\hline Liposarcoma & $2(8 \%)$ \\
\hline Myxoid liposarcoma & $1(4 \%)$ \\
\hline Round cell sarcoma & $1(4 \%)$ \\
\hline Non-typable sarcoma & $1(4 \%)$ \\
\hline \multicolumn{2}{|l|}{ Histological grade by biopsy } \\
\hline Grade 1 & $3(12 \%)$ \\
\hline Grade 2 & $1(4 \%)$ \\
\hline Grade 3 & $20(80 \%)$ \\
\hline Non-valuable & $1(4 \%)$ \\
\hline \multicolumn{2}{|l|}{ Clinical stage } \\
\hline \multicolumn{2}{|l|}{$7^{\text {th }}$ AJCC TNM staging system } \\
\hline IA & $1(4 \%)$ \\
\hline IB & $2(8 \%)$ \\
\hline$\| \mathrm{A}$ & $1(4 \%)$ \\
\hline IIB & $1(4 \%)$ \\
\hline III & $20(80 \%)$ \\
\hline IV & $0(\%)$ \\
\hline \multicolumn{2}{|l|}{$8^{\text {th }}$ AJCC TNM staging system } \\
\hline IA & $1(4 \%)$ \\
\hline IB & $2(8 \%)$ \\
\hline ॥ & $1(4 \%)$ \\
\hline IIIA & $5(20 \%)$ \\
\hline IIIB & $15(60 \%)$ \\
\hline IV & $1(4 \%)$ \\
\hline \multicolumn{2}{|l|}{ Treatment } \\
\hline $\begin{array}{l}\text { Median number of days from diagnosis } \\
\text { to onset of neoadjuvancy }\end{array}$ & $\begin{array}{c}35 \text { days } \\
(11-100)\end{array}$ \\
\hline $\begin{array}{l}\text { Median number of days from the end } \\
\text { of the neoadjuvancy to the surgery }\end{array}$ & $\begin{array}{c}46 \text { days } \\
(25-141)\end{array}$ \\
\hline Type of surgery & $22(88 \%)$ \\
\hline Excision amputation & $2(8 \%)$ \\
\hline No surgical intervention & $1(4 \%)$ \\
\hline
\end{tabular}


Table 1. Summary of the results of the study

\begin{tabular}{|c|c|}
\hline Median number of days of admission & 15 days ( $1-83)$ \\
\hline \multicolumn{2}{|l|}{ Post-surgical complications } \\
\hline Surgical wound infection & $4(16 \%)$ \\
\hline Surgical wound dehiscence & $1(4 \%)$ \\
\hline Septic shock & $1(4 \%)$ \\
\hline No post-surgical complications & $18(72 \%)$ \\
\hline Not applicable & $1(4 \%)$ \\
\hline \multicolumn{2}{|l|}{ Adjuvant treatment } \\
\hline No & $22(88 \%)$ \\
\hline Yes & $2(8 \%)$ \\
\hline No surgical intervention & $1(4 \%)$ \\
\hline \multicolumn{2}{|l|}{ Pathological results after surgery } \\
\hline \multicolumn{2}{|l|}{ Histological type of the surgical specimen } \\
\hline Pleomorphic sarcoma & $7(28 \%)$ \\
\hline Post-adjuvant sarcoma & $6(24 \%)$ \\
\hline Synovial sarcoma & $4(16 \%)$ \\
\hline Fusocellular sarcoma & $2(8 \%)$ \\
\hline Myxoid sarcoma & $1(4 \%)$ \\
\hline Liposarcoma & $1(4 \%)$ \\
\hline Pleomorphic liposarcoma & $1(4 \%)$ \\
\hline Undifferentiated chordoma & $1(4 \%)$ \\
\hline $\begin{array}{l}\text { Malignant tumour of the peripheral nerve } \\
\text { sheath }\end{array}$ & $1(4 \%)$ \\
\hline Not applicable & $1(4 \%)$ \\
\hline \multicolumn{2}{|l|}{$\begin{array}{l}\text { Histological grade of the surgical } \\
\text { specimen }\end{array}$} \\
\hline Grade 1 & $1(4 \%)$ \\
\hline Grade 2 & $4(16 \%)$ \\
\hline Grade 3 & $12(48 \%)$ \\
\hline Non-valuable & $7(28 \%)$ \\
\hline Not applicable & $1(4 \%)$ \\
\hline \multicolumn{2}{|l|}{ Necrosis of the surgical specimen } \\
\hline$<50 \%$ & $5(20 \%)$ \\
\hline $50-90 \%$ & $14(56 \%)$ \\
\hline$\geq 90 \%$ & $5(20 \%)$ \\
\hline Not applicable & $1(4 \%)$ \\
\hline \multicolumn{2}{|l|}{ Resection margins } \\
\hline Ro & $15(60 \%)$ \\
\hline $\mathrm{R} 1$ & $8(32 \%)$ \\
\hline R2 & $1(4 \%)$ \\
\hline Not applicable & $1(4 \%)$ \\
\hline \multicolumn{2}{|l|}{ Pathological stage } \\
\hline \multicolumn{2}{|l|}{$7^{\text {th }}$ AJCC TNM staging system } \\
\hline IA & $0(0 \%)$ \\
\hline IB & $1(4 \%)$ \\
\hline$\| A$ & $1(4 \%)$ \\
\hline IIB & $4(16 \%)$ \\
\hline III & $18(72 \%)$ \\
\hline IV & $0(0 \%)$ \\
\hline Not applicable & $1(4 \%)$ \\
\hline
\end{tabular}

Table 1. Summary of the results of the study

\begin{tabular}{|c|c|}
\hline \multicolumn{2}{|l|}{$8^{\text {th }}$ AJCC TNM staging system } \\
\hline IA & $0(0 \%)$ \\
\hline IB & $1(4 \%)$ \\
\hline II & $1(4 \%)$ \\
\hline IIIA & $9(36 \%)$ \\
\hline IIIB & $13(52 \%)$ \\
\hline IV & $0(0 \%)$ \\
\hline Not applicable & $1(4 \%)$ \\
\hline \multicolumn{2}{|l|}{ Stage reduction according $7^{\text {th }}$ AJCC TNM } \\
\hline Yes & $1(4 \%)$ \\
\hline No & $23(92 \%)$ \\
\hline Not applicable & $1(4 \%)$ \\
\hline \multicolumn{2}{|l|}{ Stage reduction according $8^{\text {th }}$ AJCC TNM } \\
\hline Yes & $2(8 \%)$ \\
\hline No & $22(88 \%)$ \\
\hline Not applicable & $1(4 \%)$ \\
\hline \multicolumn{2}{|l|}{ Toxicity } \\
\hline \multicolumn{2}{|l|}{ Acute toxicity } \\
\hline \multicolumn{2}{|l|}{ Dermitis } \\
\hline No dermitis & $3(12 \%)$ \\
\hline Grade 1 & $12(48 \%)$ \\
\hline Grade 2 & $4(16 \%)$ \\
\hline Grade 3 & $4(16 \%)$ \\
\hline Not applicable & $2(8 \%)$ \\
\hline \multicolumn{2}{|l|}{ Lymphedema } \\
\hline No lymphedema & $20(80 \%)$ \\
\hline Grade 1 & $1(4 \%)$ \\
\hline Grade 2 & $2(8 \%)$ \\
\hline Grade 3 & $0(0 \%)$ \\
\hline Not applicable & $2(8 \%)$ \\
\hline \multicolumn{2}{|l|}{ Chronic toxicity } \\
\hline \multicolumn{2}{|l|}{ Fibrosis } \\
\hline No fibrosis & $16(64 \%)$ \\
\hline Grade 1 & $3(12 \%)$ \\
\hline Grade 2 & $3(12 \%)$ \\
\hline Grade 3 & $0(0 \%)$ \\
\hline Not applicable & $3(12 \%)$ \\
\hline \multicolumn{2}{|l|}{ Lymphedema } \\
\hline No lymphedema & $15(60 \%)$ \\
\hline Grade 1 & $2(8 \%)$ \\
\hline Grade 2 & $5(20 \%)$ \\
\hline Grade 3 & $0(0 \%)$ \\
\hline Not applicable & $3(12 \%)$ \\
\hline \multicolumn{2}{|l|}{ Pathological fracture } \\
\hline No & 21 (84\%) \\
\hline Yes & $0(0 \%)$ \\
\hline Not applicable & $4(16 \%)$ \\
\hline \multicolumn{2}{|l|}{ Functional limitation } \\
\hline Yes & $15(60 \%)$ \\
\hline No & $6(24 \%)$ \\
\hline Not applicable & $4(16 \%)$ \\
\hline
\end{tabular}


Table 1. Summary of the results of the study

\begin{tabular}{|l|c|}
\hline Follow-up & $\begin{array}{c}24 \text { months } \\
(0-156)\end{array}$ \\
\hline Median follow-up & \\
\hline Local control & $17(68 \%)$ \\
No & $8(32 \%)$ \\
\hline Systemic control & \\
Yes & $15(60 \%)$ \\
No & $10(40 \%)$ \\
\hline Patient status & \\
Alive & $16(64 \%)$ \\
Alive free of relapse & $11(44 \%)$ \\
Alive after local relapse & $3(12 \%)$ \\
Alive after systemic relapse & $1(4 \%)$ \\
Alive after local and systemic relapse & $1(4 \%)$ \\
Dead & $9(36 \%)$ \\
Dead due to disease & $9(36 \%)$ \\
Dead for other reasons & $0(0 \%)$ \\
\hline LPFS & \\
After a 2-year follow-up & $76 \%$ \\
After a 5-year follow-up & $51.2 \%$ \\
\hline DPFS & \\
After a 2-year follow-up & $62.5 \%$ \\
After a 5-year follow-up & $50.9 \%$ \\
\hline OS & $67.2 \%$ \\
After a 2-year follow-up & $53.8 \%$ \\
After a 5-year follow-up & \\
\hline & \\
\hline
\end{tabular}

obtained: fusocellular sarcoma (36\%), synovial sarcoma (16\%), pleomorphic sarcoma (12\%), myxoid sarcoma (8\%), leiomyosarcoma (8\%), liposarcoma (8\%), myxoid liposarcoma (4\%) and round cell sarcoma (4\%), with the rest being non-typable (4\%). According to the FNCLCC classification, 84\% were oriented as high-grade tumours and $12 \%$ as low-grade tumours, with the remaining $4 \%$ not being gradable.

According to the seventh edition of the AJCC staging system, the clinical stage of the patients was as follows: IA (4\%), IB (8\%), IIA (4\%), IIB (4\%) and III (80\%). In contrast, taking into account the eighth edition of the same, the clinical staging was as described below: IA (4\%), IB (8\%), II (4\%), IIIA (20\%), IIIB (60\%) and IV (4\%).

A median of 35 days (11-100) elapsed from the date of diagnosis to the date of initiation of neoadjuvant treatment. Most patients received a total dose over PTV1 of $50 \mathrm{~Gy}$, with the exception of two: the first did not complete neoadjuvant therapy due to fistulisation and tumour bleeding, requiring amputation at the total accumulated dose of $6 \mathrm{~Gy}$; the other died in the course of neoadjuvant treatment at the total accumulated dose of $24 \mathrm{~Gy}$, in the context of rapid local progression and septic and cardiogenic shock.

For patients who completed neoadjuvant treatment, a median of 46 days (25-141 days) elapsed from the date of the end of the neoadjuvancy to the date of surgery. In most cases, an exeresis of the tumoural lesion was performed (88\%) and only two patients required amputation: one due to R0 or R1 tumour resection could not be assured, and the other one corresponded to the previously mentioned dead patient.

The median number of days at hospital after surgery was 15 (1-83). Most patients had no postoperative incidence $(72 \%)$. Of the rest of the cases, in order of frequency, the following intercurrences stand out: infection of the surgical wound (16\%), dehiscence of the surgical wound (4\%) and septic shock (4\%).

From the pathological analysis of the surgical specimen, $24 \%$ of the cases were classified as postneoadjuvant sarcoma. Of the remaining samples, the following histological subtypes were identified: pleomorphic sarcoma (28\%), synovial sarcoma (16\%), fusocellular sarcoma (8\%), myxoid sarcoma (4\%), liposarcoma (4\%), pleomorphic liposarcoma (4\%), undifferentiated chordoma (4\%) and malignant peripheral nerve sheath tumour (4\%).

According to the FNCLCC classification, 64\% were classified as high-grade tumours and $4 \%$ as low-grade tumours; in $28 \%$ of the cases the histological gradation could not be determined. Necrosis of the surgical specimen was equal to or greater than $90 \%$ in $20 \%$ of the cases. Sixty percent of the interventions were R0 resections and R1 and R2 resection margins were considered in 32\% and $4 \%$ of the cases, respectively.

The pathological stage according to the seventh edition of the AJCC followed the next distribution: IB (4\%), IIA (4\%), IIB (16\%) and III (72\%). According to the eighth edition of the same, the pathological staging was as follows: IB (4\%), II (4\%), IIIA $(36 \%)$ and IIIB (52\%). Regarding clinical staging after neoadjuvant therapy, a decrease in the stage was observed in $4 \%$ of cases considering the seventh edition of the AJCC and in $8 \%$ of cases applying the eighth edition of the same. 
A

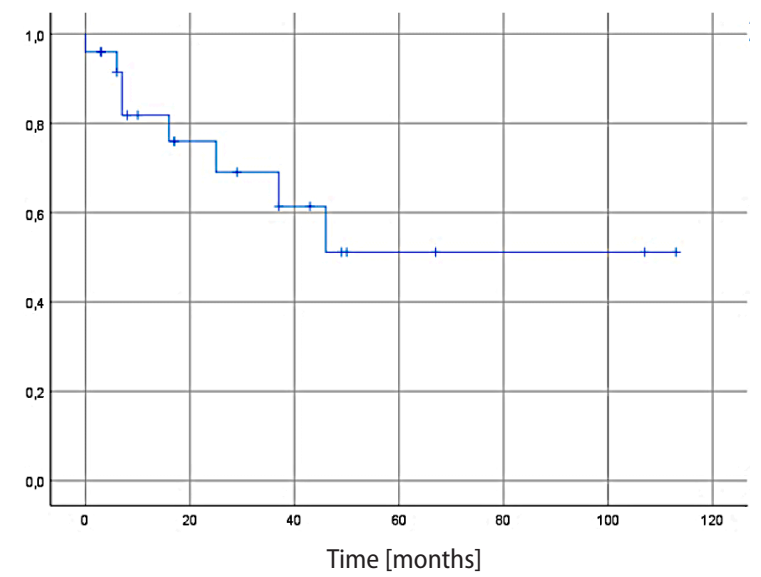

\section{B}

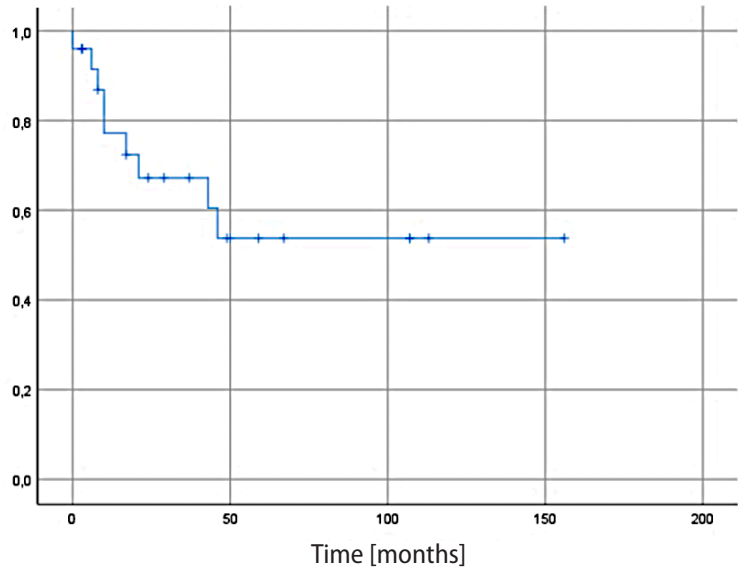

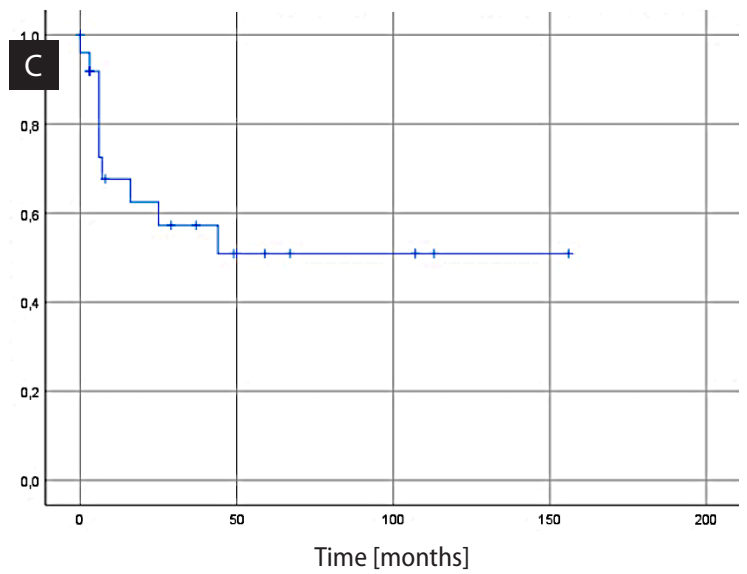

Figure 1. A. Local progression free survival (LPFS); B. Distant progression free survival (DPFS); C. Overall survival (OS)

In the cases of affected margins, additional treatment of the surgical bed with adjuvant RT was considered. This was performed in $8 \%$ of the total cases attended, with a total administered dose of 16 Gy in all cases.

Acute adverse effects identified during the course of neoadjuvant therapy and during the first month after the end of therapy were as follows: grade 1,2 and 3 dermatitis in $48 \%, 16 \%$ and another $16 \%$ of the total sample, respectively, and grade 1 and grade 2 acute lymphedema in $4 \%$ and $8 \%$, respectively, with no grade 3 events in this case. No other outstanding acute toxicities were recorded.

During the oncological monitoring, the secondaryisms exposed in the lines below were reported as chronic toxicity. Of the total sample, only $24 \%$ of the patients developed fibrosis and this was documented as grade $1(12 \%)$ and grade $2(12 \%)$, without grade 3 events. On the other hand, chronic lymphedema was described in $28 \%$ of the total sample and this was described as grade $1(8 \%)$ and grade $2(20 \%)$, also without grade 3 events. Other reported chronic toxicities were hyperchromia (8\%) and telangiectasia (4\%).

The median follow-up was 24 months (0-156 months). Local and systemic control rates were 68 and 60 percent, respectively. $64 \%$ of the patients have not died: $44 \%$ are free of relapse, $12 \%$ live after local relapse, $4 \%$ live after systemic relapse and another $4 \%$ live after local and systemic relapse. After disease progression, oncospecific treatment with systemic therapy for advanced disease was continued in most cases. The remaining 36\% have died due to disease.

Local progression free survivals (LPFS) at two and five years were $76 \%$ and $51.2 \%$ (Fig. 1A). Distant progression free survivals (DPFS) at two and five years were $62.5 \%$ and $50.9 \%$ (Fig. 1B). Finally, overall survivals (OS) at two and five years were $67.2 \%$ and $53.8 \%$ (Fig. 1C). 


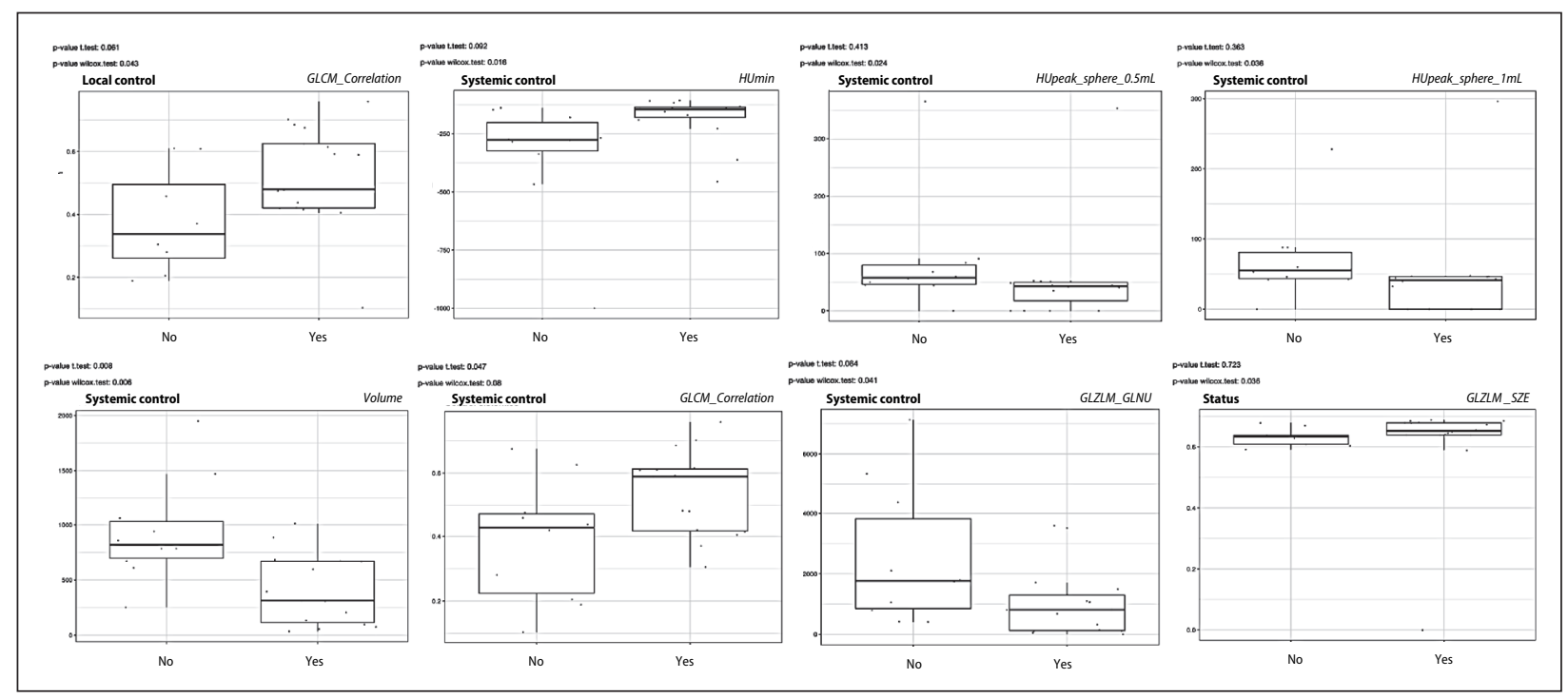

Figure 2. Randomics features analysed with significant results

After analysis of radiomics features using Student's T-test and the Wilcoxon's test considering a p-value below 0.05 to be significant, the following significant associations were found:

- local control: the variable grey level co-occurrence matrix correlation (GLCM correlation) was determined significantly by the Wilcoxon's test $(\mathrm{p}=0.043)$ (Fig. 2A);

- systemic control: the following variables were determined to be significant:

a) minimum intensity values in Hounsfield units (HUmin) using the Wilcoxon's test $(\mathrm{p}=0.016)$ (Fig. 2B),

b) medium intensity peak in Hounsfield units on a $0.5 \mathrm{~mL}$ sphere (HUpeak on a $0.5 \mathrm{~mL}$ sphere) using the Wilcoxon's test $(\mathrm{p}=0.024)$ (Fig. 2C),

c) medium intensity peak in Hounsfield units on a $0.5 \mathrm{~mL}$ sphere (HUpeak on a $1 \mathrm{~mL}$ sphere using the Wilcoxon's test $(\mathrm{p}=0.036)$ (Fig. 2D),

$\mathrm{d})$ volume using the Student's T-test $(\mathrm{p}=0.008)$ and the Wilcoxon's test $(\mathrm{p}=0.006)($ Fig. $2 \mathrm{E})$,

e) grey level co-occurrence matrix correlation (GLCM correlation) using the Student's T-test $(\mathrm{p}=0.047)$ (Fig. 2F),

f) grey level non-uniformity of grey level zone length matrix (GLZLM GLNU) using the Wilcoxon's test ( $\mathrm{p}=0.041)$ (Fig. $2 \mathrm{G}$ );

- overall survival: the variable short zone emphasis of grey level zone length matrix (GLZLM SZE) was determined significantly by the Wilcoxon's test $(\mathrm{p}=0.036)($ Fig. $2 \mathrm{H})$.

\section{Discussion}

Neoadjuvant RT is often implemented in clinical practice for the purpose of limiting the volume and dose of radiation and improving functionality because of its lower rate of fibrosis and long-term oedema. However, the current literature does not yet have conclusive data determining a prognostic benefit with respect to adjuvant RT and, therefore, data from future clinical trials are needed to determine the best treatment sequence.

There are few retrospective studies in the literature that have compared the benefit of neoadjuvant RT with adjuvant RT, with the study by Sampath et al. [7] having the largest sample size (821 patients). If we compare our analysis with the branch of neoadjuvant RT in that study (293 patients), we observe that the survival variables in our study are lower, being respectively the following: LPFS of $51.2 \% v s$. $93 \%$, DPFS of $50.9 \%$ vs. $89 \%$ and OS of $53.8 \% v s$. $65 \%$, at five years of follow- up. These differences can probably be justified by the higher number of patients compared to our analysis.

If we compare the toxicity with that observed in the phase III trial by O'Sullivan et al. [5], patients who had some complication in surgical wound healing were $20 \%$ compared to $35 \%$ in the $\mathrm{Ca}$ nadian series. Likewise, only $12 \%$ of our sample developed chronic grade 2 fibrosis without grade 3 events vs. $31.5 \%$ of the Canadian series with grade 2 events or more. Finally, chronic lymphoedema 
was observed in $20 \%$ of our sample as grade 2 without grade 3 events vs. $15.1 \%$ of the Canadian series with grade 2 events or more. Most of the RT treatments of both samples have been performed with the 3DCRT technique, so, at present, these results could probably be lower according to more recent toxicity studies with the VMAT or IMRT techniques [16].

The present study suggests a prognosis potential of CT radiomics features for risk assessment in STS. We found factors associated significantly with local control (GLCM correlation), systemic control (HUmin, HUpeak on a 0.5 and $1 \mathrm{~mL}$ spheres, volume, GLCM correlation and GLZLM GLNU) and overall survival (GLZLM SZE). In the available literature, other radiomic parameters obtained by $\mathrm{CT}$ that have shown prognostic correlation are GLRM LGRE and asymmetry (in local control, systemic and global survival) and sphericity (in local control and global survival) [17].

It should be noted that our study has a series of limitations: its retrospective nature may be a reason for potential bias, it is an exploratory phase study, its sample size is very limited and no correction has been applied for multiple comparisons (Bonferroni type).

However, the data provide a valuable basis for future research and underline the potential of Radiomics in the field of personalised medicine in STS. Future prospective trials of sufficient size may overcome the limitations of this study. Also, there would be interest in future studies to evaluate the potential prognostic characteristics of radiomics features obtained from MRI and PET images as well as the creation of nomograms that combine clinical-pathological variables and radiomics features to estimate the probability of a specific outcome.

\section{Conclusions}

After the analysis of the data obtained in this study, we can conclude that the STS treated with RT with neoadjuvant intention in our centre associate with an OS and toxicity that can be superimposed on other series. Radiomic features obtained by CT have a potential prognosis in STS risk stratification. The confection of nomograms that combine clinical-pathological variables and radiomic features could be useful in clinical practice. Our findings should be interpreted with cau- tion due to the heterogeneity of the data, their retrospective nature and the low number of patients. The results of our study may serve as a motivation for future prospective studies with a larger number of patients.

\section{Conflict of interest}

The authors declare that there is no conflict of interest.

\section{Funding}

None declared.

\section{Financial disclosure}

The authors declare that they have no relevant or material financial interests that relate to the research described in this paper.

\section{References}

1. Garcia del Muro X, de Alava E, Artigas V, et al. Spanish Group for Research on Sarcoma. Clinical practice guidelines for the diagnosis and treatment of patients with soft tissue sarcoma by the Spanish group for research in sarcomas (GEIS). Cancer Chemother Pharmacol. 2016; 77(1): 133-146, doi: 10.1007/s00280-015-2809-5, indexed in Pubmed: 26563256.

2. Burningham $Z$, Hashibe $M$, Spector $L$, et al. The epidemiology of sarcoma. Clin Sarcoma Res. 2012; 2(1): 14, doi: 10.1186/2045-3329-2-14, indexed in Pubmed: 23036164.

3. Jo VY, Fletcher CDM. WHO classification of soft tissue tumours: an update based on the 2013 (4th) edition. Pathology. 2014; 46(2): 95-104, doi: 10.1097/ PAT.0000000000000050, indexed in Pubmed: 24378391.

4. Rosenberg SA, Tepper J, Glatstein E, et al. The treatment of soft-tissue sarcomas of the extremities: prospective randomized evaluations of (1) limb-sparing surgery plus radiation therapy compared with amputation and (2) the role of adjuvant chemotherapy. Ann Surg. 1982; 196(3): 305-315, doi: 10.1097/00000658-198209000-00009, indexed in Pubmed: 7114936.

5. O'Sullivan B, Davis AM, Turcotte R, et al. Preoperative versus postoperative radiotherapy in soft-tissue sarcoma of the limbs: a randomised trial. Lancet. 2002; 359(9325): 2235-2241, doi: 10.1016/S0140-6736(02)09292-9, indexed in Pubmed: 12103287.

6. Al-Absi E, Farrokhyar F, Sharma R, et al. A systematic review and meta-analysis of oncologic outcomes of preversus postoperative radiation in localized resectable soft-tissue sarcoma. Ann Surg Oncol. 2010; 17(5): 1367-1374, doi: 10.1245/s10434-009-0885-7, indexed in Pubmed: 20217260.

7. Sampath S, Schultheiss TE, Hitchcock YJ, et al. Preoperative versus postoperative radiotherapy in soft-tissue sarcoma: multi-institutional analysis of 821 patients. Int J Radiat Oncol Biol Phys. 2011; 81(2): 498-505, doi: 10.1016/j. ijrobp.2010.06.034, indexed in Pubmed: 20888702. 
8. Alektiar KM, Brennan MF, Healey JH, et al. Impact of intensity-modulated radiation therapy on local control in primary soft-tissue sarcoma of the extremity. J Clin Oncol. 2008; 26(20): 3440-3444, doi: 10.1200/JCO.2008.16.6249, indexed in Pubmed: 18612160.

9. Callegaro D, Miceli R, Mariani L, et al. Soft tissue sarcoma nomograms and their incorporation into practice. Cancer. 2017; 123(15): 2802-2820, doi: 10.1002/cncr.30721, indexed in Pubmed: 28493287.

10. Naghavi A, Yang G, Latifi K, et al. The Future of Radiation Oncology in Soft Tissue Sarcoma. Cancer Control. 2018; 25(1): 107327481881550, doi: 10.1177/1073274818815504.

11. Tanaka K, Ozaki T. New TNM classification (AJCC eighth edition) of bone and soft tissue sarcomas: JCOG Bone and Soft Tissue Tumor Study Group. Jpn J Clin Oncol. 2019; 49(2): 103-107, doi: 10.1093/jjco/hyy157, indexed in Pubmed: 30423153.

12. Common Terminology Criteria for Adverse Events (CTCAE) v5.0. Publish Date: November 27, 2017. https:// ctep.cancer.gov/protocoldevelopment/electronic applications/docs/CTCAE_v5_Quick_Reference_5x7. pdf.
13. Nioche C, Orlhac F, Boughdad S, et al. LIFEx: A Freeware for Radiomic Feature Calculation in Multimodality Imaging to Accelerate Advances in the Characterization of Tumor Heterogeneity. Cancer Res. 2018; 78(16): 4786-4789, doi: 10.1158/0008-5472.CAN-18-0125, indexed in Pubmed: 29959149.

14. IBM Corp. IBM SPSS Statistics for Windows, Version 25.0. Released 2017.

15. PyRadiomics Community. http://github.com/radiomics/ pyradiomics.

16. O'Sullivan B, Griffin AM, Dickie $\mathrm{Cl}$, et al. Phase 2 study of preoperative image-guided intensity-modulated radiation therapy to reduce wound and combined modality morbidities in lower extremity soft tissue sarcoma. Cancer. 2013; 119(10): 1878-1884, doi: 10.1002/cncr.27951, indexed in Pubmed: 23423841.

17. Peeken JC, Bernhofer M, Spraker MB, et al. CT-based radiomic features predict tumor grading and have prognostic value in patients with soft tissue sarcomas treated with neoadjuvant radiation therapy. Radiother Oncol. 2019; 135: 187-196, doi: 10.1016/j.radonc.2019.01.004, indexed in Pubmed: 30961895. 